\title{
The roles of aromatic residues in the glycine receptor transmembrane domain
}

\author{
Bijun Tang and Sarah C. R. Lummis ${ }^{*}$ (1)
}

\begin{abstract}
Background: Cys-loop receptors play important roles in fast neuronal signal transmission. Functional receptors are pentamers, with each subunit having an extracellular, transmembrane (TM) and intracellular domain. Each TM domain contains 4 a-helices (M1-M4) joined by loops of varying lengths. Many of the amino acid residues that constitute these a-helices are hydrophobic, and there has been particular interest in aromatic residues, especially those in M4, which have the potential to contribute to the assembly and function of the receptor via a range of interactions with nearby residues.

Results: Here we show that many aromatic residues in the M1, M3 and M4 a-helices of the glycine receptor are involved in the function of the receptor. The residues were explored by creating a range of mutant receptors, characterising them using two electrode voltage clamp in Xenopus oocytes, and interpreting changes in receptor parameters using currently available structural information on the open and closed states of the receptor. For 7 residues function was ablated with an Ala substitution: 3 Tyr residues at the extracellular end of M1, 2 Trp residues located towards the centers of $\mathrm{M} 1$ and $\mathrm{M} 3$, and a Phe and a Tyr residue in M4. For many of these an alternative aromatic residue restored wild-type-like function indicating the importance of the $\pi$ ring. $\mathrm{EC}_{50} \mathrm{~s}$ were increased with Ala substitution of 8 other aromatic residues, with those in $\mathrm{M} 1$ and $\mathrm{M} 4$ also having reduced currents, indicating a role in receptor assembly. The structure shows many potential interactions with nearby residues, especially between those that form the M1/M3/M4 interface, and we identify those that are supported by the functional data.
\end{abstract}

Conclusion: The data reveal the importance and interactions of aromatic residues in the GlyR M1, M3 and M4 a-helices, many of which are essential for receptor function.

Keywords: Ligand-gated ion channel, Cys-loop receptor, Aromatic amino acid, Mutagenesis, Electrophysiology

\section{Background}

Cys-loop receptors, which include nicotinic acetylcholine (nACh), 5- $\mathrm{HT}_{3}, \mathrm{GABA}_{\mathrm{A}}$, and glycine (Gly) receptors, are pentameric ligand-gated ion channels (pLGIC) responsible for fast excitatory and inhibitory synaptic neurotransmission in the central and peripheral nervous systems [1-4]. Members of this family are pentameric, with each of the subunits having an extracellular domain (ECD), a transmembrane domain (TMD), and an intracellular domain. Molecules that activate these receptors bind at the interface between two adjacent subunits in the ECD,

*Correspondence: sl120@cam.ac.uk

Department of Biochemistry, University of Cambridge, Cambridge, UK triggering a conformational change that ultimately opens the ion channel in the TMD.

Recent high resolution structural data from both eukaryotic and prokaryotic pLGICs have begun to clarify their mechanism of action [5-12], but, as these are only snapshots in a particular state, much still remains to be discovered. The structures do, however, allow us greater insight into the specific roles of amino acids in these important proteins, which should ultimately allow us to understand how they contribute to protein function. Gly receptors (GlyR), especially $\alpha 1$ GlyR, have been particularly well examined to date. They are unusual in that they can function as homomeric proteins, thus allowing easier interpretation of functional data following mutagenesis. They also express well in heterologous systems, and 
indeed have proved to be the vertebrate pLGIC of choice for structural studies: there are more high resolution GlyR structures available than for any other Cys-loop receptor. Thus these proteins are good candidates for this type of study.

It has long been appreciated that aromatic residues are important in Cys-loop receptors, and in the GlyR many studies have demonstrated that the aromatic residues in the ECD play roles in the function, assembly, stability and expression of receptors [13-18]. Of particular importance are those aromatics clustered in the agonist or orthosteric binding site, which constitute the "aromatic box" found in all pLGIC. There are also many aromatic residues in the TMD (Fig. 1) and there have been a range of studies on these, with most of them being concerned with the residues in M4. Studies suggest that M4 interactions with M1 and M3 in some pLGICs are necessary to promote effective interactions between the $\mathrm{M} 4 \mathrm{C}$-terminus and the Cys-loop, which are located at the interface of the ECD and the TMD, and are important in coupling agonist binding to channel opening $[19,20]$. It is also possible that there are more direct links, for example W418 in the nAChR M4 $\alpha$-helix has recently been shown to interact directly with S226 on the adjacent M1 $\alpha$-helix, stabilizing the open state [21]. Thus understanding the interactions made by aromatic residues is an important step in understanding the mechanism of activation of pLGICs. Here we extend previous studies on the GlyR
TMD aromatic residues, which were performed before the structure of this protein was available, and interpret the data using structural information now available from open and closed states of the GlyR. The data show that many of these residues play important roles in the function of the receptor.

\section{Methods \\ Oocyte maintenance}

Xenopus oocytes were purchased from EcoCyte Bioscience (Dortmund, Germany) and stored in ND96 (96 mM $\mathrm{NaCl}, 2 \mathrm{mM} \mathrm{KCl}, 1.8 \mathrm{mM} \mathrm{CaCl}_{2}, 1 \mathrm{mM} \mathrm{MgCl}, 5 \mathrm{mM}$ HEPES, pH 7.5) containing $2.5 \mathrm{mM}$ sodium pyruvate, $50 \mathrm{mM}$ gentamycin and $0.7 \mathrm{mM}$ theophylline.

\section{HEK293 cell culture}

Human embryonic kidney (HEK) 293 cells (ECACC 85120602 Sigma Aldrich, UK) were maintained on $90 \mathrm{~mm}$ tissue culture plates at $37{ }^{\circ} \mathrm{C}$ and $7 \% \mathrm{CO}_{2}$ in a humidified atmosphere. They were cultured in DMEM:F12 with GlutaMAX ${ }^{\text {TM }}$ I media (Dulbecco's Modified Eagle's Medium/ Nutrient Mix F12 (1:1), Invitrogen, Paisley, UK) containing $10 \%$ foetal calf serum. For immunofluorescent studies cells on cover slips were transfected using polyethyleneimine (PEI). $30 \mu \mathrm{l}$ PEI $(1 \mathrm{mg} / \mathrm{ml}), 5 \mu \mathrm{l} \mathrm{cDNA}$ and $1 \mathrm{ml}$ DMEM were incubated for $10 \mathrm{~min}$ at room temperature, added drop wise to an $80-90 \%$ confluent plate, and incubated for $2-3$ days before use.

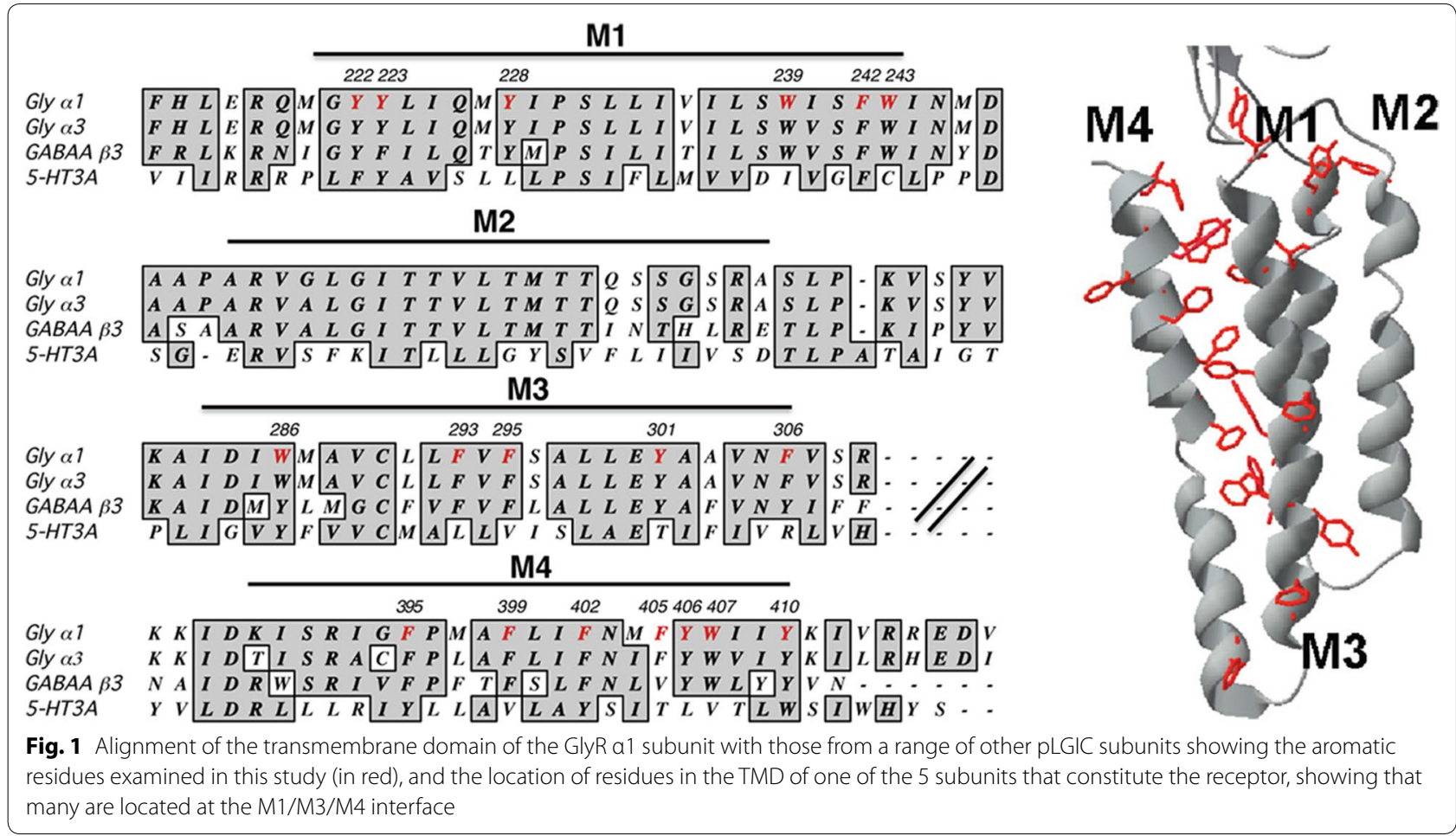




\section{Receptor expression}

cDNA was cloned into pGEMHE for oocyte expression, and pcDNA3.1 (Invitrogen, Paisley, UK) for expression in HEK 293 cells. Mutagenesis was performed using QuikChange (Agilent Technologies Inc., CA, USA); primers are shown in Additional file 1: Table S1. cRNA was in vitro transcribed from linearised pGEMHE cDNA template using the mMessage mMachine T7 Transcription kit (Ambion, Austin, TX, USA). Oocytes were injected with $50 \mathrm{nl}$ of $\sim 400 \mathrm{ng} \mathrm{ll}^{-1} \mathrm{cRNA}$, and currents were recorded 1-4 days post-injection.

\section{Electrophysiology}

This was similar to our previous work $[14,15]$ although here we used a Robocyte voltage-clamp system (Multichannel systems, Germany). Briefly Xenopus oocytes were clamped at $-60 \mathrm{mV}$, and oocytes perfused with

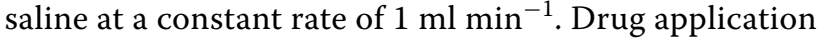
was via a simple gravity fed system calibrated to run at the same rate. Extracellular saline contained $(\mathrm{mM}), 96$ $\mathrm{NaCl}, 2 \mathrm{KCl}, 1.8 \mathrm{CaCl}_{2}, 1 \mathrm{MgCl}_{2}$ and 5 HEPES; $\mathrm{pH} 7.4$ with $\mathrm{NaOH}$ ). Analysis and curve fitting was performed using Prism v4.03 (GraphPad Software, San Diego, CA, USA, www.graphpad.com). Concentration-response data for each oocyte were normalised to the maximum current for that oocyte. Statistical significance was determined using an ANOVA with a Dunnetts multiple comparison post test.

\section{Immunocytochemistry}

This was as described previously [22]. Briefly, transiently transfected fixed cells were incubated in anti-glycine $\alpha 1$ receptor antisera $(\mathrm{C}-15$; Santa Cruz Biotechnology). Biotinylated anti-rabbit IgG (Vector Laboratories, CA, USA) and fluorescein isothiocyanate avidin D (Vector Laboratories) were then used to detect bound antibody. Immunofluorescence was observed using a Leica fluorescent microscope.

\section{Structures}

PDBs 5VDH (open GlyR), 5CFB (closed GlyR), 4COF $\left(\mathrm{GABA}_{\mathrm{A}} \mathrm{R}\right)$ and 4HIF (Gloeobacter ligand-gated ion channel, GLIC) were downloaded from the PDB database and viewed and/or mutated using PyMol or Swiss-PDBViewer. The GlyR structures are those of the homomeric $\alpha 3$ GlyR, but the majority of residues, and in particular all the aromatic residues studied here, are identical to those in the $\alpha 1$ GlyR (Fig. 1).

\section{Results}

To probe the roles of the aromatic residues in the transmembrane $\alpha$-helices of the GlyR, we mutated each to Ala, and determined changes in functional characteristics following expression in Xenopus oocytes. We also mutated some of the more sensitive residues to an alternative aromatic residue, to clarify possible interactions with the $\pi$ ring. Example concentration response curves and current traces are shown in Fig. 2. Mutation of seven of the nineteen aromatic residues to Ala resulted in nonfunctional receptors (Table 1), and, apart from one (F295A), all others resulted in increased $\mathrm{EC}_{50} \mathrm{~S}$ and/or lower maximal currents (Fig. 3), indicating these residues are important for GlyR expression and/or function. The data from each transmembrane $\alpha$-helix are discussed below.

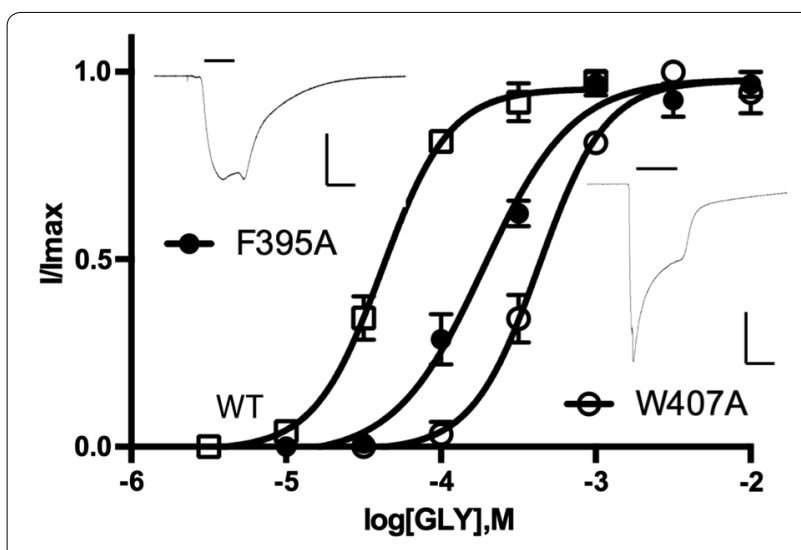

Fig. 2 Example concentration response curves and maximal current traces for WT and two mutant GlyR. Data $=$ mean \pm SEM, $n=4-8$. Parameters obtained from these curves are shown in Table 1. Scale bars are $2 \mu \mathrm{A}$ and $10 \mathrm{~s}$

Table $1 \mathrm{EC}_{50} \mathrm{~s}$ of WT and mutant GlyR

\begin{tabular}{|c|c|c|c|c|c|}
\hline Mutant & $\mathrm{pEC}_{50}(\mathrm{M})$ & $\mathrm{EC}_{50}(\mu \mathrm{M})$ & Mutant & $p E C_{50}(M)$ & $E C_{50}(\mu \mathrm{M})$ \\
\hline \multirow[t]{2}{*}{ WT } & $4.380 \pm 0.04$ & 42 & F293A & $3.559 \pm 0.06^{*}$ & 276 \\
\hline & & & F293Y & $3.901 \pm 0.02^{*}$ & 125 \\
\hline M1 & & & F295A & $4.339 \pm 0.07$ & 46 \\
\hline Y222A & NR & & Y301A & $3.463 \pm 0.09^{*}$ & 345 \\
\hline Y222F & $3.519 \pm 0.03^{*}$ & 303 & F306A & $3.795 \pm 0.03^{*}$ & 160 \\
\hline Y223A & NR & & & & \\
\hline Y223F & $3.721 \pm 0.07^{*}$ & 190 & M4 & & \\
\hline Y228A & NR & & F395A & $3.724 \pm 0.05^{*}$ & 189 \\
\hline Y228F & $4.466 \pm 0.07$ & 34 & F399A & NR & \\
\hline W239A & NR & & F399Y & $3.846 \pm 0.04^{*}$ & 143 \\
\hline W239F & $4.248 \pm 0.04$ & 57 & $\mathrm{~F} 402 \mathrm{~A}$ & $4.183 \pm 0.03$ & 66 \\
\hline F242A & $3.803 \pm 0.07^{*}$ & 157 & $\mathrm{~F} 402 \mathrm{Y}$ & $3.725 \pm 0.01^{*}$ & 188 \\
\hline \multirow[t]{2}{*}{ W243A } & $3.764 \pm 0.06^{*}$ & 172 & $\mathrm{~F} 405 \mathrm{~A}$ & $2.961 \pm 0.07^{*}$ & 1095 \\
\hline & & & F405Y & $3.718 \pm 0.07^{*}$ & 192 \\
\hline M3 & & & Y406A & $N R$ & \\
\hline W286A & NR & & W407A & $3.366 \pm 0.04^{*}$ & 430 \\
\hline W286Y & $2.351 \pm 0.05^{*}$ & 445 & Y410A & $3.489 \pm 0.16^{*}$ & 324 \\
\hline
\end{tabular}

Data $=$ mean $\pm S E M, n=3-8 ;{ }^{*}=$ significantly different to WT, $\mathrm{p}<0.05$ ANOVA with Dunnetts multiple comparison post-test; $N R=$ non-responsive 


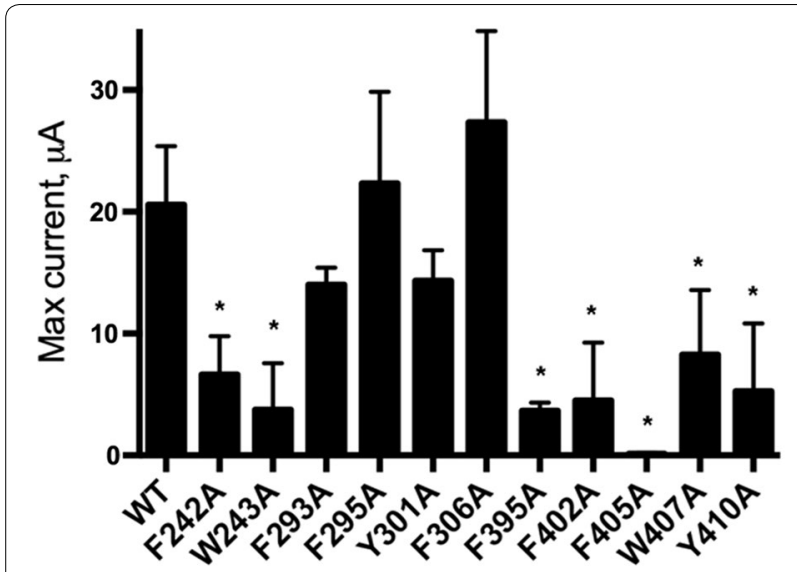

Fig. 3 Maximal currents elicited by glycine are lower in mutants with Ala substitutions to aromatics in M1 and M4. Data = mean \pm SEM, $n=4-8,{ }^{*}=$ significantly different to $W T, p<0.05$, ANOVA with Dunnetts post-comparison test

\section{Aromatic residues in $\mathbf{M} 1$}

The aromatic residues at the top of the M1 $\alpha$-helix are more sensitive than those towards the intracellular side: Y222 and Y223 at the extracellular side result in nonfunctional receptors when substituted with Ala, despite being expressed (Additional file 2: Figure S1). When these Tyrs are replaced with an alternative aromatic residue, function is retained, but with increased $\mathrm{EC}_{50} \mathrm{~S}$ (Table 1). Structural data show Y223 could interact with many neighbouring residues, although Y222 probably only with Y223 (Fig. 4).

Y228 and W239, which are towards the centre of M1, also result on non-functional receptors when substituted with Ala, but here an alternative aromatic results in $\mathrm{EC}_{50}$ values similar to wild type receptors. A number of interactions with neighbouring residues are revealed from the structural data (Figs. 5, 6, 7).

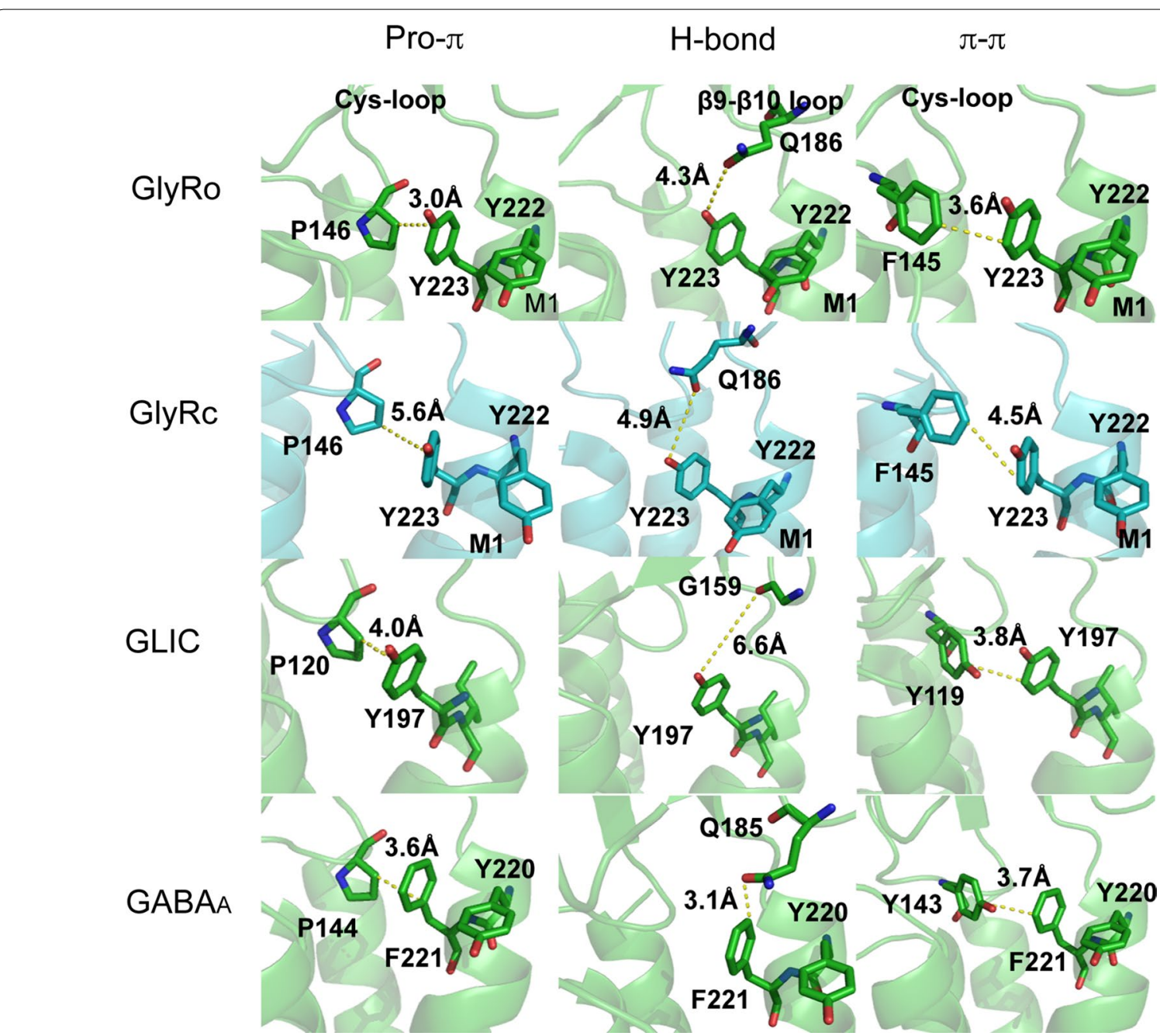

Fig. 4 Structures of Gly, GLIC and GABA receptors showing possible interactions of Y222 and Y223 or equivalent residues. GlyRo = GlyR in open state; $\mathrm{GlyRc}=\mathrm{GlyR}$ in closed state 

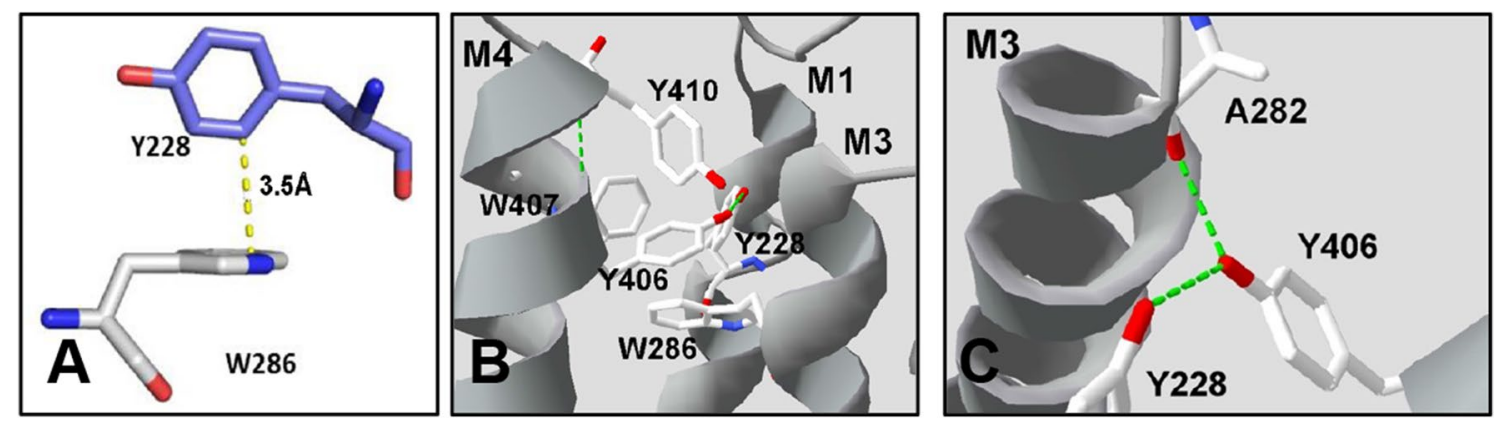

Fig. 5 A Y228 and W286 are well positioned to form a T-type $\pi-\pi$ interaction; B These residues, with Y406,W407 and Y410, form an aromatic cluster at the top of the M1/M3/M4 interface. CY406 could form a hydrogen bond (green dashes) with either A282 or Y228; the data better support the former
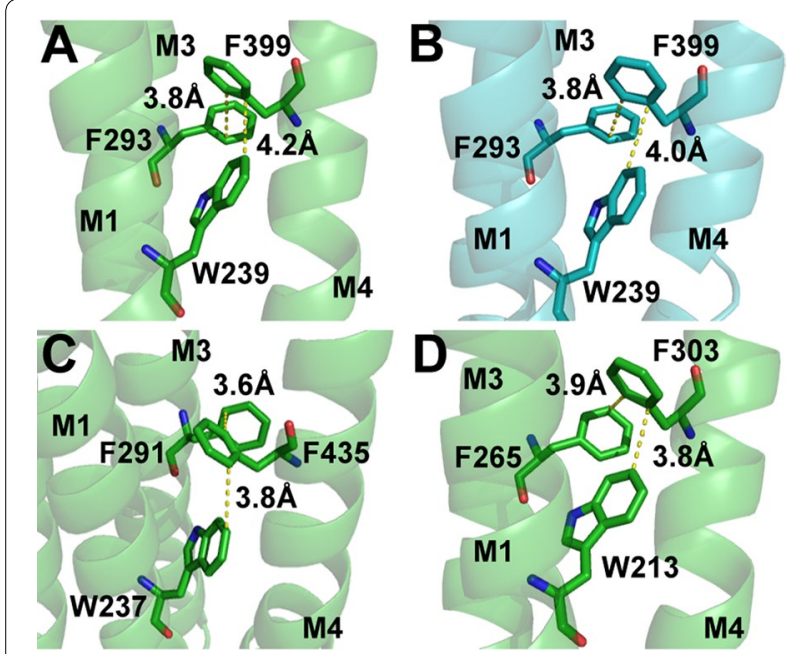

Fig. 6 GlyR in the open (A) and closed (B) states show similar interactions between W239, F293 and F399. Structural data show equivalent residue interactions are possible in the $\mathrm{GABA}_{\mathrm{A}}$ receptor $(\mathbf{C})$ and GLIC (D)

F242 and W243 differ from the other M1 aromatics as they do result in functional receptors when substituted with Ala, but both have increased $\mathrm{EC}_{50} \mathrm{~s}$ values (Table 1), and decreased maximal responses (Fig. 3), indicating that a residue with a $\pi$ ring is not essential at these locations, although it is preferred. Possible interactions with F395 and R392 respectively are revealed from the structural data (Figs. 7, 8, 9).

\section{Aromatic residues in $\mathrm{M} 3$}

Only one aromatic residue in M3 (W286) resulted in non-functional receptors when substituted with Ala, although substitution with an alternative aromatic here yielded receptors with a large $(\sim 10$ fold $)$ increase in $\mathrm{EC}_{50}$ indicating the importance of this Trp. Ala substitution of 3 of the 4 other aromatic residues in this loop

resulted in increases in $\mathrm{EC}_{50}$ (3-8 fold), while F295Acontaining receptors were wild type-like (Table 1 ). Thus again these data suggest that a residue with a $\pi$ ring is favored (apart from at position 295), but not essential. All of these receptors had maximal currents that were similar to wild type (Fig. 3). Various potential interactions of these M3 aromatics were observed from the structural data (Figs. 5, 6, 7, 8, 9).

\section{Aromatic residues in M4}

Ala substitution of the aromatic residues in M4 have been previously reported [17], and our data are similar, revealing non-functional receptors with F399A and F406A-containing receptors, and increases in $\mathrm{EC}_{50}$ for most of the others, showing the impact of the $\pi$ ring. 

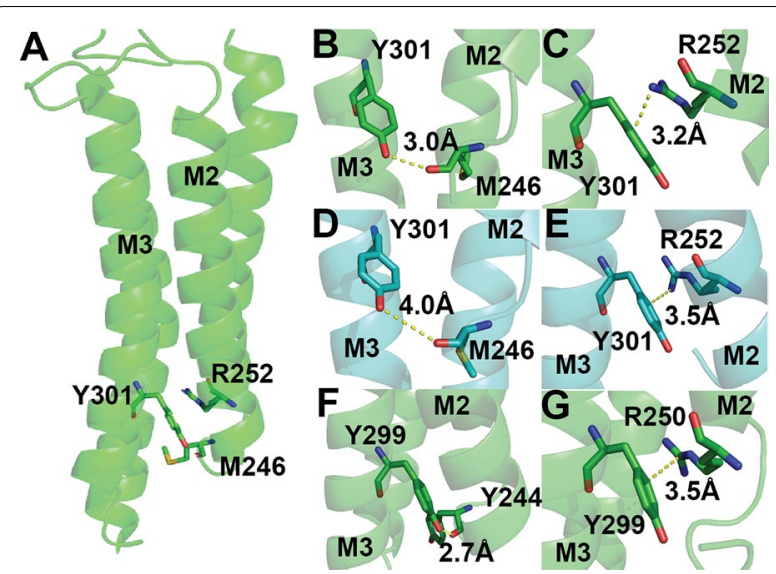

Fig. 8 Y301 in M3 (A) could form a hydrogen bond with M246 and/ or a cation- $\pi$ interaction with $\mathrm{R} 252$ in the open state $(\mathbf{B}, \mathbf{C})$ but probably not the former in the closed state $(\mathbf{D}, \mathbf{E})$. Similar interactions are possible in the $\mathrm{GABA}_{\mathrm{A}}$ receptor $(\mathbf{F}, \mathbf{G})$

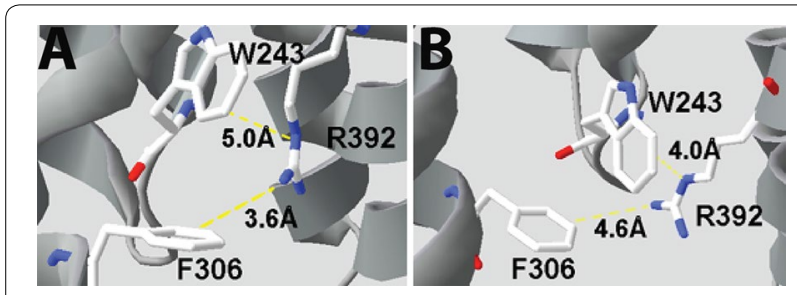

Fig. 9 F306 could form a cation- $\pi$ interaction with R392 in the open state (A), but R392 is more likely to do this with W243 in the closed state (B)

All functional receptors also had decreased maximal currents compared to wild-type receptors (Fig. 3). To further explore some of these we substituted an alternative aromatic, which for some partially restored function (Table 1). F402 was unusual here in that substitution with Ala resulted in wild-type-like receptors but with Tyr resulted in an increased $\mathrm{EC}_{50}$. Interaction of these residues with many of their neighbours was observed from the structural data (Figs. 5, 6, 7, 8).

\section{Discussion}

In order to understand the roles of individual residues in receptors, it is critical to combine both structural and functional data. Here we have used such data to understand the interactions and importance of aromatic residues in the GlyR TMD. Aromatic residues are among many hydrophobic residues located, as would be expected, in the transmembrane $\alpha$-helices, and many of these hydrophobic residues play some role in the assembly and/or correct function of the TMD.
Aromatic residues, however, are especially important, as they have the potential for more interactions with adjacent residues because of their $\pi$ rings. Indeed we observed that these residues frequently contribute to aromatic networks that exist between the different $\alpha$-helices that constitute the TMD, and many are necessary for the efficient functioning of the protein; these interactions and networks are discussed in more detail below.

\section{Y222 and Y223}

Y222 and Y223 are located at the N-terminus of the M1 $\alpha$-helix of the GlyR. Our data indicate both these residues are important for receptor function, with Ala substitution ablating function, although this is retained, albeit with an increase in $\mathrm{EC}_{50}$, with a Phe substitution. Our data do differ from a previous study [17] who found that Ala substitution did not ablate function. We cannot currently explain this, although in that study currents were reduced (e.g. Y223A currents were $14 \%$ of WT) and no $\mathrm{EC}_{50}$ data were given, so it is difficult to directly compare them with the current work. We did, however, observe that both Y222A and Y223A-containing GlyR were expressed in HEK293 cells, suggesting aromatic residues at these locations are not essential for receptor expression.

The structural data indicate that the only interaction that Y222 could make with adjacent residues is a $\pi-\pi$ interaction with Y223. Such an interaction could help stabilise Y223, which has the potential for multiple interactions as shown in Fig. 4. Firstly it could form a bond between the aromatic ring of Y223 and the polarized $\mathrm{CH}$ in P146 (a CH- $\pi$ bond) in the Cys-loop. A Pro in the Cys-loop is essential for pLGIC function, and it has been proposed this family of proteins should be renamed Pro-loop receptors because of the conservation of this Pro in all pLGICs [23]. Previous studies suggest this Pro may form a critical cis peptide bond [24, 25]; the intrinsically higher cis bias of Pro peptide bonds compared to other residues is consistent with this proposal. It is possible that a $\mathrm{CH}-\pi$ bond here could assist in stabilizing a cis peptide bond, and/or this interaction could hold the Cys-loop in a position or orientation that allows gating: the distance between Y223 and P146 differs in the open and closed states, and thus an interaction here might be important to stabilize the GlyR in the open state. GLIC and $\mathrm{GABA}_{\mathrm{A}} \mathrm{R}$ structures show similar distances between equivalent residues, indicating that similar interactions may occur in these and perhaps all pLGICs.

Y223 could also form a hydrogen bond with Q186, located on the $\beta 9-\beta 10$ loop, another important region for channel gating (Fig. 4). However, the distance between these residues suggest a hydrogen bond here would be weak, and it is not conserved in GLIC and $\mathrm{GABA}_{\mathrm{A}} \mathrm{R}$. 
In addition the $\mathrm{EC}_{50}$ difference when the $\mathrm{OH}$ in Tyr is removed (i.e. in Phe) is small ( $<4$ fold). Thus we conclude there is no hydrogen bond here.

Y223 is also an appropriate distance and orientation from F145 in the Cys-loop to form a $\pi-\pi$ interaction (Fig. 4). F145 in the GlyR has been shown to be important in providing a hydrophobic framework for a strong electrostatic interaction between D148 in the Cys-loop and R218 in M1 [26], and an interaction with Y223 could assist in the correct positioning of F145. Conservation of similar residues between GlyR, GLIC and GABA ${ }_{A} R$ provide some support for this hypothesis, although the distances observed suggest that this interaction is less likely than that with P146, although both could contribute to Cys-loop stability.

\section{Y228, W286, Y406, W407 and Y410}

Y228 and W286 are located at the N-terminal end of the M1 $\alpha$-helix and M3 $\alpha$-helix respectively, and substitution with Ala ablates function, consistent with previous studies that show no surface expression [17], while substitution with an alternative aromatic (Phe or Tyr respectively) results in receptors that function well (Table 1). These data suggest that $\pi$ rings are important in residues at these locations for receptor assembly, and the structure reveals Y228 and W286 are ideally placed to form a T-type $\pi-\pi$ interaction (Fig. 5A). Replacement of Y228 with Phe would have little effect on such an interaction, consistent with a WT-like $\mathrm{EC}_{50}$ of Y228F mutant GlyR, while a W286 to Tyr substitution would have a greater impact, as the orientations of the $2 \pi$ rings would likely be less than ideal; our data showing an $\sim 10$ fold increase in $\mathrm{EC}_{50}$ in W286Y-containing GlyR are again consistent with this interpretation. These residues are not, however, the only aromatics in this location: Y406, W407 and Y410 at the C-terminal end of the M4 $\alpha$-helix are close to Y228 and W286, and these five residues form an aromatic cluster between the M1, M3 and M4 $\alpha$-helices at the extracellular side of the TMD (Fig. 5B). The loss of Gly-elicited responses in Y406A-containing GlyR, consistent with previous studies that show no surface expression [17], and increase in $\mathrm{EC}_{50}$ in W407A-and Y410A-containing GlyR, supports contributions of these aromatic residues to the assembly and function of the receptor as previously proposed [17]. These authors also demonstrated that these latter residues contribute to assembly as shown by their lower expression levels (and resulting lower maximal currents) with Ala substitution, and our data is similar, supporting this proposal (Fig. 3). They also suggested that Y228 interacts with F293, F402 and F405, but we now know that Y228 is too far from these residues for any interaction. Similarly Y406, W407 and Y410 were aberrantly suggested to face and interact with the lipid bilayer, stabilizing the M4 $\alpha$-helix. The structure reveals they actually face the interface of M1, M3 and M4, and thus do likely have a role in stabilizing M4, but by interacting with M1 and M3 $\alpha$-helices and not the lipid.

The structural data also predict a hydrogen bond between Y228 and Y406, but the WT-like response of Y228F containing GlyR indicates that if there is a hydrogen bond here it does not contribute to receptor function. Y406 could alternatively hydrogen bond with the backbone of A282 (Fig. 5C) and, given the increase in $\mathrm{EC}_{50}$ with Y406F containing GlyR, we propose this interaction does occur, and has a role in linking M1 and M4 that is perhaps important for assembly.

These suggested interactions are supported by studies in the $\mathrm{GABA}_{\mathrm{A}} \mathrm{R}$, where $\mathrm{Y} 474$ (equivalent to $\mathrm{Y} 406$ ) is predicted to hydrogen bond with Y289 (Y228), and Y474 has a likely $\pi-\pi$ interaction with Y346 (W286) on M3 [27]. In GLIC, these aromatic residues are not conserved. However, I202 (Y228), I259 (W286), L310 (Y406) and A311 (W407) could perhaps form a hydrophobic patch that plays a similar role.

\section{W239, F293 and F399}

These residues are located in the M1/M3/M4 interface just below the aromatic cluster described above. Mutation of W239 and F399 to Ala ablated function, consistent with previous studies that show no surface expression [17], while function was retained with an alternative aromatic (Phe or Tyr respectively; Table 1), indicating a residue with a $\pi$ ring is necessary for assembly. An aromatic at F293 is less important: F293A containing GlyR had an $\sim 4$ fold increase in $\mathrm{EC}_{50}$ which was reduced to $\sim 2$ fold with a Tyr substitution, suggesting that a small aromatic residue is preferred but not essential. We propose these residues interact with each other via $\pi-\pi$ interactions (Fig. 6), which plays a role in stabilizing the transmembrane domain of the glycine receptor. Haeger et al. [17] also suggested there are interactions here, but details were inaccurate due to the lack of structural data. Such interactions are further supported by GLIC and GABA receptor structural data: the Phe equivalent to F399 in M4 could interact with the Trp equivalent of W239 in M1 and the Phe equivalent of F293 in M3.

\section{W239, F242 and F395}

These residues on M1 (W239 and F242) and M4 (F395) could form $\pi-\pi$ interactions (Fig. 7A, B) with W239 linking this group of aromatic residues with the one described above. W239 is the most sensitive of these residues to Ala substitution, where function is ablated as described above, whereas there are $\mathrm{EC}_{50} \mathrm{~s}$ increases of 3-4 fold and decreases in maximal currents (as 
previously reported [17]) for F242A and F395A-containing GlyR (Table 1, Fig. 3) indicating roles in both assembly and function. WT-like responses with W239F containing GlyR suggest an aromatic residue is important here, and the altered distance between this residue and F395 in the open and closed states of the receptor suggest a $\pi-\pi$ interaction that plays a role in stabilizing the open state. Data from other pLGIC indicate the equivalent residues in GLIC F216(M1) and F299(M4), ELIC F222(M1) and F303(M4) and GABApR F303 (M1) and F463 (M4) may have similar interactions (e.g. Fig. 7C, D).

\section{Y301, F306 and W243}

These residues are located towards the intracellular side of the TMD. Y301 and F306 are on M3 and have not been previously studied. Our data indicate they play a role in function but not expression, as Ala substitutions resulted in changes to $\mathrm{EC}_{50} \mathrm{~s}$ (Table 1) but not $\mathrm{I}_{\max }$ (Fig. 3), while W243 on M1, where Ala substitution results in changes to both $\mathrm{EC}_{50}$ and $\mathrm{I}_{\max }$ could have roles in both expression and function.

Ala substitution of Y301 results in an 8 fold increase in $\mathrm{EC}_{50}$. This residue has the potential to hydrogen bond with the backbone of M246 on M2 and/or form a cation- $\pi$ interaction with R252, also on M2 (Fig. 8AE). The distance between Y301 and M246 varies by $1 \AA$ between the open and closed states, whereas the distance between Y301 and R252 is similar. These data suggest a possible role of the hydrogen bond in stabilising the open state, while the cation $-\pi$ interaction links M2 or M3, which could be important for information transfer between the transmembrane $\alpha$-helices. Equivalent residues in the $\mathrm{GABA}_{\mathrm{A}}$ receptor could also form both the hydrogen bond and the cation $-\pi$ interaction (Fig. 8F, G), providing some support for our suggestion, although no equivalent aromatic residue is present in GLIC.

Ala substitution of F306 or W243 both result in an $\sim 4$ fold increase in $\mathrm{EC}_{50}$. F306 is sufficiently close to R392 (M4) to form a cation- $\pi$ interaction in the open but not the closed state, while W243 could form a cation $-\pi$ interaction in the closed but not the open state (Fig. 9); such interactions could play a role in receptor opening and/or closing. Phe and $\operatorname{Trp}$ residues are conserved at these locations in the $\mathrm{GABA}_{\mathrm{A}}$ receptor and GLIC, supporting this hypothesis. W243 is also less than $4 \AA$ from the backbone of W239, and thus could help stabilize this residue, which, as discussed above, is important for receptor expression.

\section{F295 and F405}

Ala substitution of F295 and F405 produce receptors that are similar to WT, and the structural data reveal both these residues face away from the core of the protein into the lipid bilayer. This indicates, as expected, that any hydrophobic residue could likely be located at these positions, as the presence of a $\pi$ ring is not necessary.

\section{Conclusions}

In conclusion we have used functional data combined with structural information to reveal the importance and interactions of the aromatic residues in the TMD of the glycine receptor. Some of these residues, especially those in M4, have been previously studied, but without the structural data it was previously not possible to accurately determine how they might interact with adjacent residues. The new information we have provided allow the clarification of the roles of these residues, and will also contribute to a full understanding of the mechanism of action of these critical neuronal proteins.

\section{Additional files}

Additional file 1: Table S1. Oligonucleotides used to create the Ala mutants of each transmembrane aromatic residue.

Additional file 2: Figure S1. Example immunofluorescent images from WT F222A, and F223A GlyR shows all were expressed in HEK293 cells.

\section{Abbreviations}

nACh: nicotinic acetylcholine; pLGIC: pentameric ligand-gated ion channels; ECD: extracellular domain; TMD: transmembrane domain; GlyR: glycine receptor; DMEM: Dulbecco's Modified Eagle's Medium; HEK: human embryonic kidney; GLIC: gloeobacter ligand-gated ion channel.

Authors' contributions

BT contributed to data acquisition and data analyses. SCRL contributed to the experimental design, data acquisition, data analyses and manuscript writing. Both authors read and approved the final manuscript.

\section{Acknowledgements}

We thank Jenny Jeffreys for expert technical assistance.

\section{Competing interests}

The authors declare that they have no competing interests.

Availability of data

All analysed data is presented in the manuscript. Raw datasets can be obtained from the corresponding author upon reasonable request.

\section{Consent for publication}

Not applicable.

Ethics approval and consent to participate Not applicable.

Funding

SCRL was supported by an MRC Grant (MR/L021676).

\section{Publisher's Note}

Springer Nature remains neutral with regard to jurisdictional claims in published maps and institutional affiliations. 
Received: 4 July 2018 Accepted: 29 August 2018

Published online: 06 September 2018

\section{References}

1. Burgos CF, Yevenes GE, Aguayo LG. Structure and pharmacologic modulation of inhibitory glycine receptors. Mol Pharmacol. 2016;90(3):318-25.

2. Lynch JW. Molecular structure and function of the glycine receptor chloride channel. Physiol Rev. 2004;84(4):1051-95.

3. Lynch JW. Native glycine receptor subtypes and their physiological roles. Neuropharmacology. 2009;56(1):303-9.

4. Thompson AJ, Lester HA, Lummis SC. The structural basis of function in Cys-loop receptors. Q Rev Biophys. 2010;43(4):449-99.

5. Bocquet N, Nury H, Baaden M, Le Poupon C, Changeux JP, Delarue M, Corringer PJ. X-ray structure of a pentameric ligand-gated ion channel in an apparently open conformation. Nature. 2009;457(7225):111-4.

6. Hassaine G, Deluz C, Grasso L, Wyss R, Tol MB, Hovius R, Graff A, Stahlberg $H$, Tomizaki T, Desmyter A, et al. X-ray structure of the mouse serotonin 5-HT3 receptor. Nature. 2014;512(7514):276-81.

7. Hibbs RE, Gouaux E. Principles of activation and permeation in an anionselective Cys-loop receptor. Nature. 2011;474(7349):54-60.

8. Hilf RJ, Dutzler R. X-ray structure of a prokaryotic pentameric ligand-gated ion channel. Nature. 2008:452(7185):375-9.

9. Hilf RJ, Dutzler R. Structure of a potentially open state of a proton-activated pentameric ligand-gated ion channel. Nature. 2009;457(7225):115-8

10. Huang $X$, Chen H, Michelsen $K$, Schneider S, Shaffer PL. Crystal structure of human glycine receptor-alpha3 bound to antagonist strychnine. Nature. 2015;526(7572):277-80.

11. Huang $X$, Shaffer $P L$, Ayube $S$, Bregman $H$, Chen H, Lehto SG, Luther JA, Matson DJ, McDonough SI, Michelsen K, et al. Crystal structures of human glycine receptor alpha3 bound to a novel class of analgesic potentiators. Nat Struct Mol Biol. 2017;24(2):108-13.

12. Miller PS, Aricescu AR. Crystal structure of a human GABAA receptor. Nature. 2014;512(7514):270-5

13. Schofield CM, Trudell JR, Harrison NL. Alanine-scanning mutagenesis in the signature disulfide loop of the glycine receptor alpha 1 subunit: critical residues for activation and modulation. Biochemistry. 2004:43(31):10058-63.

14. Pless SA, Millen KS, Hanek AP, Lynch JW, Lester HA, Lummis SC, Dougherty DA. A cation-pi interaction in the binding site of the glycine receptor is mediated by a phenylalanine residue. J Neurosci. 2008;28(43):10937-42.
15. Pless SA, Hanek AP, Price KL, Lynch JW, Lester HA, Dougherty DA, Lummis SC. A cation-pi interaction at a phenylalanine residue in the glycine receptor binding site is conserved for different agonists. Mol Pharmacol. 2011;79(4):742-8

16. Grudzinska J, Schemm R, Haeger S, Nicke A, Schmalzing G, Betz H, Laube $B$. The beta subunit determines the ligand binding properties of synaptic glycine receptors. Neuron. 2005;45(5):727-39.

17. Haeger S, Kuzmin D, Detro-Dassen S, Lang N, Kilb M, Tsetlin V, Betz H, Laube B, Schmalzing G. An intramembrane aromatic network determines pentameric assembly of Cys-loop receptors. Nat Struct Mol Biol. 2010;17(1):90-8.

18. Braun N, Lynagh T, Yu R, Biggin PC, Pless SA. Role of an absolutely conserved tryptophan pair in the extracellular domain of Cys-loop receptors. ACS Chem Neurosci. 2016:7(3):339-48.

19. Henault CM, Juranka PF, Baenziger JE. The M4 transmembrane alphahelix contributes differently to both the maturation and function of two prokaryotic pentameric ligand-gated ion channels. J Biol Chem. 2015;290(41):25118-28.

20. daCosta CJ, Dey L, Therien JP, Baenziger JE. A distinct mechanism for activating uncoupled nicotinic acetylcholine receptors. Nat Chem Biol. 2013;9(11):701-7

21. Domville JA, Baenziger JE. An allosteric link connecting the lipid-protein interface to the gating of the nicotinic acetylcholine receptor. Sci Rep. 2018;8(1):3898.

22. Reeves DC, Lummis SC. Detection of human and rodent 5-HT3B receptor subunits by anti-peptide polyclonal antibodies. BMC Neurosci. 2006;7:27.

23. Jaiteh M, Taly A, Henin J. Evolution of pentameric ligand-gated ion channels: pro-loop receptors. PLoS ONE. 2016;11(3):e0151934.

24. Deane CM, Lummis SC. The role and predicted propensity of conserved proline residues in the 5-HT3 receptor. J Biol Chem. 2001:276(41):37962-6.

25. Limapichat W, Lester HA, Dougherty DA. Chemical scale studies of the Phe-Pro conserved motif in the cys loop of Cys loop receptors. J Biol Chem. 2010;285(12):8976-84

26. Pless SA, Leung AW, Galpin JD, Ahern CA. Contributions of conserved residues at the gating interface of glycine receptors. J Biol Chem. 2011;286(40):35129-36

27. Cory-Wright J, Alqazzaz M, Wroe F, Jeffreys J, Zhou L, Lummis SCR. Aromatic residues in the fourth transmembrane-spanning helix M4 are important for GABA $\rho$ receptor function. ACS Chem Neurosci. 2018;9(2):284-90
Ready to submit your research? Choose BMC and benefit from:

- fast, convenient online submission

- thorough peer review by experienced researchers in your field

- rapid publication on acceptance

- support for research data, including large and complex data types

- gold Open Access which fosters wider collaboration and increased citations

- maximum visibility for your research: over 100M website views per year

At BMC, research is always in progress.

Learn more biomedcentral.com/submissions 